

\title{
Análise da elasticidade de transmissão de preços externos no mercado doméstico da soja maranhense
}

\author{
Analysis of the elasticity of transmission \\ of external prices in the domestic market \\ of soy from Maranhão
}

\author{
Lindalva Silva Correia* | Sergiany da Silva Lima**
}

\begin{abstract}
Resumo
O trabalho analisa a elasticidade de transmissão de preços do mercado internacional de soja para o mercado de soja do Maranhão, inspirado em Mundlack e Larson (1992). Para estimar a transmissão de preços restrita a Lei do Preço Único, é utilizado o estimador Vetor de Correção de Erros (VEC), sem e com restrição. As séries temporais de preços se mostraram cointegradas, sugerindo a existência de relações de longo prazo entre os mercados. $\mathrm{O}$ teste de causalidade Granger sugere que há causalidade unidirecional dos preços internacionais da soja cotada na Bolsa de Chicago sobre os preços da soja maranhense. As elasticidades estimadas através do VEC não rejeitam a restrição imposta de transferência integral dos preços da soja internacional no longo prazo. Os choques de preços externos não anunciados são transferidos para o preço da soja maranhense em uma intensidade que cresce até o terceiro mês, e estabilizam em 15 meses.
\end{abstract}

Palavras-chave: Lei do Preço Único; Vetor de Correção de Erro; Cadeia de Comercialização.

\begin{abstract}
The paper analyzes the price transmission elasticity of the international soybean market to the Maranhão soybean market, inspired by Mundlack and Larson (1992). To estimate the price transmission restricted by the Single Price Law, the Error Correction Vector (VEC) estimator is used, without and with restriction. Time series of prices have been co-integrated, suggesting the existence of long-term relationships between markets. The Granger causality test suggests that there is unidirectional causality in international soybean prices quoted on the Chicago Stock Exchange over the prices of Maranhão soy. The elasticities estimated through the VEC do not reject the restriction imposed on the full transfer of international soybean prices in the long run. Unannounced external price shocks are transferred to the price of Maranhão soy in an intensity that grows until the third month and stabilizes in 15 months.
\end{abstract}

Keywords: Single Price Law; Error Correction Vector; Chain Trading.

\footnotetext{
* Professora Adjunta do Departamento de Economia da Universidade Federal do Maranhão. https://orcid.org/0000-0002-3719-0149. E-mail: lindacmaia@bol.com.br

** Professor Adjunto da Unidade Acadêmica da Universidade Federal Rural de Pernambuco. https://orcid.org/0000-0003-3606-7971. E-mail: sergiany.lima@ufrpe.br
} 


\section{INTRODUÇÃO}

O conhecimento da transmissão de preços entre os mercados é de grande valor na elaboração de estratégias de comercialização dos produtores, a exemplo da elaboração dos contratos de comercialização e de políticas públicas. Por essa razão, é possível ver diversos estudos sobre transmissão de preços no mercado de commodities, nas últimas duas décadas. No Brasil, há uma forte expansão desse tipo de investigação especialmente a partir dos anos 2000. O conhecimento das relaçóes de preços entre os mercados assume uma importância considerável nas relaçóes comerciais, sobretudo na elaboração dos contratos de comercialização, assim como na implantação de políticas agrícolas direcionada à cadeia produtiva (ADAMI; MIRANDA, 2011).

Atualmente o Maranhão configura-se como importante fronteira agrícola que compreende o bioma do Cerrado MATOPIBA, composto pelos estados do Maranhão, Tocantins, Piauí e Bahia. A produção de soja no estado começa em 1990, com 15.305 ha de terra plantada, especialmente na mesorregião sul. Entre 1991 e 1992 a área plantada com soja cresce 360,7\% no estado (IBGE, 2016). A Lei Kandir de 13 de setembro de 1996 teve efeitos diretos sobre os produtos in natura em todo o país, com a desoneração fiscal sobre as exportações, incentivando as exportações de soja in natura. Até 1997, a produção de soja ainda era restrita quase exclusivamente ao sul maranhense. Em 2001 a produção de soja alcança uma área total de 213.436 ha, abrangendo as mesorregiôes leste e oeste do Maranhão. Em 2015, o estado já possui uma área total plantada de 761.225 mil ha, quase o triplo de 1996.

Atualmente, o estado do Maranhão caracteriza-se como o oitavo maior produtor de soja do Brasil e segundo maior da Região Nordeste (IBGE, 2016). Com exceção da mesorregião norte, que é composta pela Aglomeração Urbana de São Luís, Baixada Maranhense, Itapecuru Mirim, Lençóis Maranhenses e Rosário, todas as demais produzem soja. O estado apresenta vantagens competitivas por estar integrado aos grandes mercados consumidores, a exemplo da China. O baixo custo das suas terras ${ }^{1}$ e a proximidade com o porto do Itaqui constituem características estratégicas para região, integrando o comércio aos mercados da América do Norte e Europa. A inserção da soja na mesorregião sul do estado, sobretudo nos Gerais de Balsas, se deu em princípio devido ao baixo preço da terra e políticas públicas de incentivo, reforçadas pelas melhorias de infraestrutura logística e programas de apoio ao agronegócio. As condições geográficas, logísticas, e edafoclimáticas favorecem a cadeia produtiva da soja no estado (CUNHA, 2015).

Entre 2010 a 2014, o crescimento da produção de soja no valor adicionado da produção agrícola foi de aproximadamente 30,43\%, alcançando uma participação de 3\% da produção agrícola (IMESC, 2016). Desse modo, 
o objetivo deste exercício, consiste em analisar a elasticidade de transmissão de preços no mercado da soja maranhense, a partir dos preços praticados na Bolsa de Valores de Chicago e da taxa de câmbio nominal. Em outras palavras, o que se quer é identificar a estabilidade entre os preços da soja nacional e internacional; determinar a causalidade direcional entre os preços da soja maranhense e internacional no mercado de comodities; e estimar a elasticidade de transmissão dos preços irrestrito e restrito a Lei do Preço Único. A contribuição dessa pesquisa consiste em entender a formação de preços do mercado maranhense de soja, que até então se mostra preterida a discussão empírica nacional de transmissão de preços.

\section{REFERENCIAL TEÓRICO}

\section{Teoria da transmissão de preços}

$\mathrm{Na}$ análise da relação matemática entre variáveis econômicas, a elasticidade de transmissão de preços tem um significado importante. Matematicamente é semelhante a qualquer tipo de elasticidade, porém, conceitualmente, determina a transmissão de preços entre mercados. A transmissão de preços pode ser vertical ou horizontal. A transferência de preços é vertical quando existe sensibilidade entre os preços de um mercado para outro. A transferência de preços é horizontal quando existe sensibilidade entre os preços do mesmo produto em diferentes mercados. A elasticidade horizontal é também chamada de elasticidade espacial (BARROS; BURNQUIST, 1987 apud MARGARIDO, 2012).

$\mathrm{O}$ poder de mercado na atividade agrícola moderna tem se tornado matéria de recorrente preocupação entre produtores e governo, como sugere a Legislação Antitruste. Os impactos do poder de mercado têm exercido fortes efeitos na distribuição do retorno dos ganhos gerados na cadeia de comercialização. A transmissão assimétrica de preços beneficia grupos específicos dentro da cadeia de comercialização em detrimento de outros. Com a transmissão simétrica de preços, os ganhos ocorrem mais cedo e/ou em uma magnitude maior para todos os agentes envolvidos. Dessa forma, a monopolização do mercado de commodities nacionais pode reduzir fortemente os ganhos dos menores integrantes da cadeia de mercados. A Transmissão Assimétrica de Preços (ATP) pode gerar grandes efeitos no bem-estar dos agentes, com implicações sociais e políticas. Por essa razão, a minimização das assimetrias de mercado tem a função de reduzir as desigualdades nos ganhos produtivos e as vantagens individuais. A assimetria na transmissão de preços afeta o tempo e a intensidade do bem-estar dos agentes. A antecipação do tempo e a ampliação da intensidade na transmissão dos preços representa 
uma forma de vantagem conferida pela assimetria do poder de mercado (MEYER; TAUBADEL, 2004).

A transmissão assimétrica dos preços pode ser classificada de três formas: pela velocidade ou magnitude da transmissão assimétrica do preço; pelos efeitos positivos ou negativos sobre os ganhos; e pela transmissão assimétrica de natureza vertical ou horizontal. A assimetria na transmissão de preços pode estar relacionada à intervenção política, assimetria de informações, de gestão, e poder de mercado. No entanto, entende-se que os mercados não competitivos e o custo de ajustes são as causas mais relevantes da assimetria na transmissão de preços (MEYER; TAUBADEL, 2004). Em geral, os estudos que buscam avaliar as relaçóes entre os preços nos diferentes estágios de um sistema agroindustrial, seja assimetria vertical ou horizontal, procuram respostas para três questões principais: identificar a origem das variaçóes dos preços, o sentido do efeito desses choques sobre os demais preços da cadeia de comércio, e a intensidade da transmissão dos choques (MAYORGA et al., 2007); (AGUIAR; FIGUEIREDO, 2011 apud FIGUEIREDO; SOUZA FILHO; PAULLILO, 2013).

Quando as elasticidades de transmissão de preços são diferentes de 1, pode sugerir que haja uma falha de mercado. Essa falha pode estar associada ao poder de mercado do comprador, ou assimetria na transmissão dos preços com perda de bem-estar para algum elo da cadeia. Por outro lado, se a elasticidade é igual à unidade, significa que os preços estão sendo transmitidos na mesma proporção de sua alteração. Nesse caso, há perfeita transmissão de preços que pode ser um bom indicativo de uma distribuição equitativa de ganhos entre os agentes. No entanto, pode ser que haja elasticidade unitária, mas que na verdade oculta compensaçóes por uso de diferentes matérias-primas, que sobem, descem e se compensam (GUANZIROLI et al., 2008).

\section{Revisão empírica}

Adami; Miranda (2011) analisam a dinâmica da formação de preços no mercado nacional de arroz em casca, para os mercados produtores do Rio Grande do Sul (RS) e Mato Grosso (MT) no período de 2004 a 2010. O seu objetivo é definir o mercado dominante na formação de preços e os mercados seguidores, através da análise de transmissão de preços. O resultado do teste de causalidade de Granger aponta que os preços no RS são importantes para prever os preços em MT. O modelo de transferência estimado com correção de erro (VEC) mostra que, para cada $1 \%$ de aumento na taxa de crescimento dos preços no RS, a taxa de crescimento dos preços em MT registra, em média, aumento contemporâneo de $0,44 \%$. 
Margarido (2012) analisa a integração do mercado internacional de grãos de soja, considerando o comportamento dos preços dessa commodity no Brasil, na Argentina e no mercado de Rotterdam no período de outubro de 1998 a dezembro de 2009. Para esse estudo, utiliza-se o modelo de transmissão espacial de preços desenvolvido por Ravallion (1986). Os resultados apontam que os mercados do grão de soja no Brasil e em Rotterdam são plenamente integrados, significando que, no longo prazo, os preços nos mercados, interno e externo, tendem a igualar-se, confirmando a hipótese inerente a Lei do Preço Único.

Brum et al. (2015) buscam identificar a presença de cointegração e a influencia cambial nos preços nas cidades de diferentes regióes produtoras no interior do país. Os resultados encontrados indicam que, apesar das diferenças de curto prazo entre os preços, eles são cointegrados no longo prazo. Não é identificada uma influência estatística da taxa de câmbio sobre os preços da soja doméstica nas cidades de Sorriso (MT), Rondonópolis (MT), Maringá (PR), Passo Fundo (RS), Mogiana (SP) e Uberlândia (MG). Ao contrário disso, há uma relação pouco relevante quanto à transmissão de alguns impulsos sistêmicos que ajudam a explicar o comportamento dos preços no curto prazo, sem efeitos duradouros sobre estes.

Silva et al. (2017) investigam as relações de longo prazo da transmissão de preços da soja produzida no Mato Grosso, Paraná e Rio Grande do Sul, restrita a condição de Lei do Preço Único, entre janeiro de 2005 a dezembro de 2015. Entre as principais conclusões entende-se que as variaçốes no preço da soja no Paraná são transmitidas integralmente para o Mato Grosso e Rio Grande do Sul. Os coeficientes de correção de erro apontam que os preços da soja se ajustam rapidamente a desequilíbrios de curto prazo nos preços nacionais desta commodity. Os resultados indicam mercados perfeitamente integrados no longo prazo, aceitando a hipótese da Lei de Preço Único no mercado internacional da soja doméstica.

Presotto et al. (2018) analisam a transmissão do preço pago ao produtor de soja brasileiro nas praças de Passo Fundo (RS), Maringá (PR) e Rondonópolis (MT), de 1997 a 2016. A análise é realizada através de um VEC, discutido a luz da hipótese da Lei do Preço Único. Entre os principais resultados, destaca-se a significância estatística da transmissão de preços da Chicago Board of Trade (CBOT), seguido da taxa de câmbio e do prêmio de exportação. As variáveis da análise se mostram cointegradas no longo prazo. A elasticidade de transmissão de preços no longo prazo próxima de uma, sugere uma possível transferência integral dos preços da Chicago Board of Trade.

Larre (2019) estuda a integração da soja modificada e não modificada geneticamente, no mercado internacional de 1999 a 2019. Utilizando os 
preços FOB dos principais exportadores da soja modificada (Estados Unidos e Argentina) e da soja não modificada (Brasil), testa a cointegração dos mercados e a causalidade de Granger. Assume a hipótese de integração, mesmo com as regulamentações contra a soja modificada nos seus principais destinos. Todos os testes mostram que o mercado entre os três exportadores permanece integrado. Os testes de causalidade de Granger mostram que os EUA continuam sendo o único líder em preços. As elasticidades de curto prazo da transmissão de preços para o Brasil e Argentina são respectivamente de $0,33 \%$ e $0,25 \%$. Os resultados aceitam a Lei do Preço Único de que há transmissão integral dos preços Americanos no longo prazo.

Copetti; Coronel (2020) examinam o efeito denominado pass-through da taxa de câmbio, Real/Dólar (R\$/US\$) - Real/Euro (R\$/€), nos preços de exportação da soja brasileira, de janeiro de 2000 a dezembro de 2018. Os resultados indicam que o grau de pass-through da taxa de câmbio para os preços de exportação da soja nacional é baixo, com os coeficientes negativos de -0,29 e -0,33 para o cambio em dólar e em euro, respectivamente. Devido ao baixo efeito cambial, choques cambiais nos preços da soja brasileira, não se traduzem em ganhos significativos de competitividade, dado que não reduzem expressivamente os preços de exportação.

\section{METODOLOGIA}

Modelo teórico

O modelo teórico utilizado neste trabalho é desenvolvido por Mundlack; Larson (1992), o qual se baseia na Lei do Preço Único (LPU). O referido modelo mostra como variaçóes nos preços externos afetam os preços internos. A Lei do Preço Único estabelece que, em mercados concorrenciais livres de custos de transportes e de barreiras oficiais ao comércio, bens idênticos transacionados em diferentes regióes devem ser vendidos pelo mesmo preço, quando cotados em uma mesma moeda (KRUGMAN, 2010). Isso significa que na ausência de uma intervenção, espera-se que preços no mercado interno desses produtos variam de acordo com os preços mundiais (MUNDLACK; LARSON, 1992). Desse modo, espera-se que variaçóes de preços no mercado externo sejam transmitidas, proporcionalmente, aos preços praticados no mercado doméstico. A Lei do Preço Único está diretamente relacionada ao processo de arbitragem de preços entre regióes no longo prazo (MARGARIDO et al., 2001; MARGARIDO, 2012).

O modelo de Mundlak; Larson (1992) admite que o preço doméstico de determinado produto possa ser descrito como função do seu próprio preço no exterior, dada a taxa nominal de câmbio, e a política comercial adotada ${ }^{2}$. 
Nesse modelo, a equação é baseada no pressuposto de que a taxa de câmbio não é sobrevalorizada, ou seja, diferenças entre taxas de inflação doméstica e externa estão totalmente refletidas na taxa de cambio (E). Matematicamente esta função pode ser escrita como:

$$
P_{t i}^{R \$}=P_{t i}^{U \$} E_{t}
$$

Em que $P_{t i}^{R \$}$ representa o preço interno do produto $i$ no período $t$, cotado em moeda local; $P_{t i}^{U \$}$ é o preço externo do produto $i$ no período $t$, cotado em moeda externa; e $E_{t}$ é a taxa nominal de câmbio no período $t$. Mudanças nos preços relativos da equação 1 são incorporados a mudanças cambiais. A versão relativa da teoria da paridade do poder de compra demonstra que diferenças entre as taxas de inflação doméstica $\left(\Delta P_{t i}^{R \$}\right)$ e internacional $\left(\Delta P_{t i}^{U \$}\right)$ são captadas por mudanças na taxa nominal de cambio $\left(\Delta E_{t}\right)$.

$$
\Delta E_{t}=\Delta P_{t i}^{R \$}-\Delta P_{t i}^{U \$}
$$

Modelo empírico

Assumindo a forma funcional de determinação do preço doméstico através da Lei do Preço Único, é possível descrever a função econométrica de transmissão dos preços dos grãos de soja maranhense com a seguinte modelagem empírica:

$$
P_{t}=\left(P_{t}^{*}\right)^{\beta_{1}}\left(E_{t}\right)^{\beta_{2}} u_{t}
$$

O termo $u_{t}$ é um distúrbio aleatório com distribuição independente e identicamente distribuída, com média $\mu$ e variância constante $u_{t} \sim I I D\left(\mu, \sigma^{2}\right)$. Dentro de um modelo $\log$-log os coeficientes $\beta_{1}$ e $\beta_{2}$ representam as elasticidades das relações funcionais. Assim, se a elasticidade é igual a unidade $\left(\beta_{1}=1\right)$, significa dizer que os preços estão sendo transmitidos na mesma proporção de sua alteração original ou plenamente transmitidos. Tal transmissão representa um indicativo de uma distribuição eficiente de ganhos proporcionados pelo aumento do preço do produto na cadeia de valor. Ao contrário, se o valor de $\beta$, é igual a zero $\left(\beta_{1}=0\right)$ significa que não há relacionamento entre os mercados, tratando-se, assim, de uma economia autárquica.

Tomando o logaritmo da equação 3 , tem-se a representação $\left(P_{t}\right)$ empírica da equação econométrica de transmissão dos preços domésticos do mercado dos grãos de soja maranhenses, em relação aos preços externos $\left(P_{t}^{*}\right)$ da commodity, e ao preço da moeda brasileira no mercado cambial nacional $\left(E_{t}\right)$. 


$$
\ln P_{t}=\beta_{0}+\beta_{1} \ln P_{t}^{*}+\beta_{2} \ln E_{t}+\vartheta_{t} ; \operatorname{com} \vartheta_{t}=\ln u_{t} ; \vartheta_{t} \sim I I D\left(\mu, \sigma^{2}\right)
$$

$\mathrm{O}$ caso mais comum demonstra que o valor de $\beta$, varia entre zero e um $\left(0 \leq \beta_{1} \leq 1\right)$, refletindo a omissão de variáveis importantes, como é o caso mais típico da política comercial adotada pelo país ou ainda qualquer outro tipo de restrição imposta ao mercado. A elasticidade menor que 1 (um) demonstra uma transmissão imperfeita de preços (MUNDLACK; LARSON, 1992). O coeficiente $\beta_{2}$ representa a elasticidade da taxa de câmbio nominal, indicando como variaçốes na taxa de câmbio são transferidas para os preços internos. Parte-se da hipótese de que, variações na taxa de câmbio são integralmente transmitidas aos preços domésticos no longo prazo. Logo, espera-se que $\beta$, também assuma valor igual à unidade, tal como no trabalho desenvolvido por Margarido (2012).

\section{Apresentação das variáveis e fonte dos dados}

Neste exercício são utilizadas três séries de tempo com observações referentes às variáveis: preços do grão da soja no Maranhão, preços do grão da soja no mercado externo, e a taxa de câmbio nominal. O preço doméstico da soja no Maranhão $(P)$ tem como proxy o preço do grão de soja ao produtor, disponível no site Agrolink ${ }^{3}$. Para deflação do preço doméstico, é utilizado o Índice Geral de Preços da Fundação Getúlio Vargas (FGV/Conj. Econ. IGP), a preços constantes de junho de 2009 . O preço externo da soja $\left(P^{*}\right)$, tem como proxy os preços da commoditie cotados em Chicago Board of Trade ${ }^{4}$ (CBOT), em dólar americano (US\$) por tonelada métrica, disponível no site do Fundo Monetário Internacional (FMI). Esse preço foi convertido para $\mathrm{R} \$$ por saca de 60 quilogramas. Para deflacionar a série de preço externo, é utilizado o índice de preços do Federal Reserve Bank of St. Louis, com preço constante de junho de 2009. A taxa de câmbio nominal $(E)$ tem como proxy a taxa de câmbio anual determinada pelo site do Banco Central do Brasil, seção Balanço de Pagamentos. O resumo da fonte dos dados está disponibilizado no Quadro 1 abaixo.

Quadro 1- Descrição das séries de tempo e das variáveis da pesquisa

\begin{tabular}{|l|l|l|l|}
\hline Variáveis & Definição das variáveis & Fonte & Tempo \\
\hline$P$ & $\begin{array}{l}\text { Preço ao Produtor do grão de soja } \\
\text { Maranhense }\end{array}$ & Agrolink & Abril de 2009 a fevereiro de 2016 \\
\hline$P^{*}$ & $\begin{array}{l}\text { Preço Externo cotado na Bolsa de } \\
\text { Chicago Board of Trade }\end{array}$ & FMI/IFS & Abril de 2009 a fevereiro de 2016 \\
\hline$E$ & Taxa nominal de câmbio & BACEN & Abril de 2009 a fevereiro de 2016 \\
\hline
\end{tabular}

Fonte: Elaboração própria com dados secundários da CBOT; AGROLINK; e BACEN (2016). 
Os dados utilizados neste exercício compreendem o período de abril de 2009 a fevereiro de 2016, gerando um total de 83 observaçóes. O motivo da opção por esse período justifica-se pela indisponibilidade de dados contínuos de preços da soja no Maranhão. Somente a partir desse período é que as séries de preços não apresentaram interrupções. Para realização do ajuste sazonal ou a dessazonalização ${ }^{5}$ das séries, empregou-se o método X-12 ARIMA, implementado pelo U.S. Bureau of the Census.

\section{Modelo econométrico}

O método econométrico empregado na estimação das elasticidades de transmissão de preços é o Vetor de Correção de Erros (VEC). A sua função é medir o efeito das transmissóes de preços considerando um modelo irrestrito, e outro restrito. No modelo irrestrito, é estimado um VEC de transmissão de preços sem condicionar as elasticidades de curto prazo aos vetores cointegrados de perfeita transmissão dos preços na cadeia de comércio, como sugere a Lei do Preço Único. No modelo restrito, o VEC é estimado impondo a restrição de perfeita transmissão de preços. Esses dois exercícios econométricos podem ser utilizados para entender o modelo mais ajustado sobre a transmissão de preços para o mercado da soja produzida no estado do Maranhão. O mesmo procedimento é realizado por Mundlack; Larson (1992); e Margarido et al. (2001).

A estimação de Vetores de Correção de Erros pressupõe duas condiçốes estatísticas básicas das séries temporais: precisam possuir a mesma ordem de integração e serem cointegradas. Se as séries são cointegradas, significa que há uma estabilidade de longo prazo entre as séries. Contudo, a cointegração depende da ordem de integração das séries. Portanto, antes da estimação da equação de transmissão de preços são realizados os testes de raiz unitária de Dickey-Fuller Aumentado (ADF), e de cointegração de Johansen. Como se trata de um estudo de transmissão de preços externos sobre o preço da soja maranhense, é realizado também o teste de causalidade Granger para determinar o sentido direcional da causalidade entre os preços.

\subsubsection{Raiz unitária}

Para determinar a ordem de integração das variáveis de interesse, este trabalho utiliza o teste de raiz unitária Dickey-Fuller Aumentado (ADF) que permite identificar a ordem de integração de uma série. $\mathrm{O}$ teste consiste na estimação dos parâmetros de um modelo autorregressivo (GUJARATI; PORTER, 2011).

$$
y_{t}=\rho y_{t-1}+u_{t} ; \quad u_{t}=\text { é um erro ruído branco }
$$


Se $\rho=1$, pode se dizer que há raiz unitária. Entretanto, a estimativa dessa equação por Minimos Quadrados Ordinários (MQO) é extremamente viesada, na presença de raíz unitária. Dessa forma, somando um zero a equação autoregressiva da raiz unitária é possível chegar a mesma equação em primeira diferença.

$$
\Delta y_{t}=\delta y_{t-1}+u_{t}
$$

Sendo que $\Delta$ é um operador de primeira diferença, e $\delta=\rho-1$. A estimação de $\delta$ sob a hipótese nula $\delta=0$, implica dizer que se essa hipótese for aceita, entende-se que há raiz unitária, porque para $\delta=0$, necessariamente $\rho=1$.

Dickey e Fuller conseguem demonstrar que sob a hipótese nula de $\delta=0$, a estatística da $t$ do teste de hipótese do teste de hipótese do parâmetro estimado possui uma distribuição (tau). Portanto, na literatura esse teste passou a ser chamado de Dickey-Fuller (DF). O teste Dickey-Fuller é estimado sob três diferentes hipóteses nulas, em que:

A raiz unitária é descrita por um passeio aleatório

$$
\Delta y_{t}=\delta y_{t-1}+u_{t} ; \quad \Delta y_{t}=u_{t}
$$

A raiz unitária é descrita é um passeio aleatório com deslocamento;

$$
\Delta y_{t}=\beta_{1}+\delta y_{t-1}+u_{t} ; \quad \Delta y_{t}=\beta_{1}+u_{t}
$$

A raiz unitária é um passeio aleatório com deslocamento em torno de uma tendência determinística

$$
\Delta y_{t}=\beta_{1}+\beta_{2} t+\delta y_{t-1}+u_{t} ; \quad \Delta y_{t}=\beta_{1}+\beta_{2} t+u_{t}
$$

Essas três equações do teste DF assumem que o termo de erro $\left(u_{t}\right)$ sejam não correlacionados. Para os casos em que o $u_{t}$ são autocorrelacionados, os referidos autores desenvolveram o teste Dickey-Fuller aumentado (DFA). Essa é uma extensão das equaçóes anteriores, adicionando como independente a variável dependente defasada.

$$
\Delta y_{t}=\beta_{1}+\beta_{2} t+\sum_{i=1}^{m} \quad \alpha_{i} \Delta y_{t-i}+\delta y_{t-1}+u_{t}
$$

Nos três casos, se a hipótese nula for rejeitada, significa dizer que a série é estacionaria, possivelmente em tono de uma tendência determinística. Nesse caso, se diz que a série é estacionária ou é integrada de ordem zero (I(0)). Se for aceita a hipótese nula de que há raiz unitária corrigida por uma diferença, se diz que a série é não estacionária integrada de ordem um (I(1)). O número de diferenças para tornar-se uma série estacionária determina a sua ordem de integração. De maneira genérica, se uma série é estacionaria com diferenças, 
se diz que a série é integrada de ordem $d(\mathrm{I}(d))$. Para todo $d>0$ a estimação de qualquer modelo com séries temporal vai depender da análise de cointegração, ou estabilidade de longo prazo.

\subsubsection{Análise de Cointegração}

O teste de cointegração de Johansen oferece uma conclusão estatística sobre a estabilidade de longo prazo das series temporais. A metodologia de cointegração desenvolvida por Johansen $(1991$; 1995) é descrita considerando um Vetor Autorregressivo (VAR) de ordem $p$.

$$
y_{t}=A_{1} y_{t-1}+\cdots+A_{p} y_{t-p}+B x_{t}+\epsilon_{t}
$$

Em que $y_{t}$ é um $k$-vetor não estacionário I(1), $x_{t}$ é um $d$-vetor determinístico, e $\epsilon_{t}$ é um vetor de inovação. Transformando em somatório, a equação VAR pode ser reescrita como segue:

$$
\begin{aligned}
& y_{t}=\Pi y_{t-1}+\sum_{i=1}^{p-1} \quad \Gamma_{i} \Delta y_{t-i}+B x_{t}+\epsilon_{t}, \operatorname{com} \Pi=\sum_{i=1}^{p} \quad A_{i}-I, \mathrm{e} \\
& i=-\sum_{j=i+1}^{p} \quad A_{j}
\end{aligned}
$$

Baseado na representação do teorema de Granger, se a matriz coeficiente $\Pi$ reduzida tiver ranking $r<k$, então existe $k \times r$ matrizes $\alpha e \beta$, cada uma com ranking $r$ tal que $\Pi=\alpha \beta$ e $\beta$ `y $y_{t}$ sejam $\mathrm{I}(0)$. $r$ é o número de relações de cointegração e cada coluna $\beta$ é um vetor de cointegração.

Com informaçóes a priori sobre a ordem de integração das series temporais, é recomendado o teste de especificação tendência determinística, para determinar o número de vetores de cointegração condicionais as suposiçóes feitas sobre a tendência das séries com procedimentos sequenciais de $r=0 \mathrm{e}$ $r=k-1$ (JOHANSEN, 1995). O resultado do procedimento sequencial é relatado através das estatísticas Trace e Max. Tanto a estatística Trace quanto Max, testam a hipótese nula de ausência cointegração contra a hipótese alternativa de cointegração dos vetores $r$.

\subsubsection{Análise de Causalidade}

O teste de causalidade busca verificar se a incorporação de valores defasados de uma variável $x$ contribui com melhores previsóes para outra variável $y$. Nesse sentido, a identificação de uma causalidade sugere o sentido em que a transmissão de preços ocorre. Assim, trata-se de um teste de precedência temporal e não de causalidade no sentido de uma relação de causa e efeito. Em outros termos, esse instrumental é útil para avaliar se variações de preços em um mercado precedem as variaçóes de preços em outro mercado (GRANGER, 1969 apud ENDERS, 2004). 
O teste de causalidade de Granger assume que os melhores previsores de um conjunto de variáveis preditivas sejam as próprias variáveis temporalmente defasadas. Nesses termos, sejam quaisquer duas variáveis $x$ e $y$, as suas melhores estimativas dessas variáveis são:

$$
\begin{array}{lll}
y_{t}=\sum_{i=1}^{n} & \propto_{i} x_{t-i}+\sum_{j=1}^{n} & \beta_{j} y_{t-j}+u_{1 t} \\
x_{t}=\sum_{i=1}^{n} & \lambda_{i} x_{t-i}+\sum_{j=1}^{n} & \delta_{j} y_{t-j}+u_{2 t}
\end{array}
$$

Como se trata de um modelo autoregressivo, é possível assumir que os termos de erro sejam não autocorrelacionados. Essas duas regressões assumem a hipótese de causalidade bilateral em que $x$ causa $y$ e $y$ causa $x$. Contudo, se apenas $x$ causar $y$, ou se apenas $y$ causar $x$, se diz que há uma causalidade unidirecional no sentido Granger. Em último caso, se o conjunto de coeficientes estimados de $x$ e $y$ for estatisticamente diferente de zero, se diz que há uma causalidade bilateral. O mesmo exercício pode ser estendido para um número maior de variáveis (GUJARATI; PORTER, 2011).

\subsubsection{Vetor de Correção de Erro}

$\mathrm{O}$ Vetor de Correção Erro (VEC) se mostra adequado para essa análise por dois motivos: modela os efeitos de curto e longo prazo da transmissão de preços; e testa a hipótese da Lei do Preço Único, através da restrição de transferência integral dos preços. O VEC é definido a partir de um Vetor Autorregressivo (VAR) $z$ irrestrito com $k$ defasagens $z_{t}=A_{1} z_{t-1}+\cdots+A_{k} z_{t-k}+\Phi D+u_{t}$, em que $z_{t}$ é um vetor $(n \times 1), A$ é uma matriz de parâmetros com ordem $(n \times n), D$ são termos determinísticos, e $u$ um distúrbio aleatório ruído branco (HARRIS, 1995). A estimação de vetores autorregressivos cointegrados pressupóe um nível ótimo de defasagens $k$ que torna o erro aleatório estacionário. Essa defasagem é determinada através dos critérios de informação: Erro de predição final (EPF); Akaike (AIC); Schwarz (SC); e Hannan-Quinn (HQ).

Como o VEC estima parâmetros de curto e longo prazo, é possível transformar um VAR I(1), em um VEC acrescentando os efeitos de curto prazo através do operador de diferença aos efeitos de longo prazo do vetor cointegrado. O modelo do vetor de correção de erro (MVEC) é, portanto, descrito da seguinte forma:

$$
\Delta z_{t}=\Gamma_{1} \Delta z_{t-1}+\cdots+\Gamma_{k-1} \Delta z_{t-k+1}+\Pi z_{t-1}+\Phi D+u_{t}
$$

Em que $\Gamma_{i}=-\left(I-A_{1}-\cdots-A_{i}\right) ; i=(1, \ldots, k-1)$; е $\Pi=-\left(I-A_{1}-\right.$ $\cdots-A_{k}$ ). O posto $r$ da matriz $\Pi$ determina o número de combinaçóes estacionárias do vetor $z$. Desse modo, para todo $0<r<p$ existem $r$ vetores cointegrados, determinando uma matriz $\Pi=\alpha \beta$, cujo $\beta$ é a matriz de 
vetores cointegrados, ou efeitos de longo prazo, e $\beta$ é a matriz de coeficientes de ajustamentos, ou efeitos de curto prazo (HARRIS, 1995).

Para verificar a hipótese da Lei do Preço Único com transferência integral do preço externo, é estimado um VEC restrito. A restrição imposta ao modelo é determinada sobre os parâmetros $\left(\beta_{i j}\right)$ de longo prazo, aonde o parâmetro estimado $\beta_{1 j}$ do preço doméstico da equação empírica $(\mathrm{P})$, é igual ao negativo dos parâmetros estimado $\beta_{2 j}$ do preço externo $\left(P^{*}\right)$ e do parâmetro estimado $\beta_{3 j}$ da taxa de cambio nominal $(E)$. A restrição atende a seguinte especificação devido a todas estarem do mesmo lado da igualdade do vetor de correção de erros $\left(\beta_{1 j}=-\beta_{2 j}=-\beta_{3 j}\right)$. Em termos matriciais, a hipótese nula $\left(b_{0}\right)$ é $\beta=H \varphi$, aonde $H$ é matriz coluna de restrição e $\varphi$ é um vetor de parâmetros a ser estimado. A rejeição de que $\varphi=0$ significa que a restrição imposta é significativa, e que há transmissão integral dos preços.

$$
H=[1-1-1]
$$

\section{RESULTADOS E DISCUSSÃO}

Taxa de câmbio, e os preços da soja no Maranhão e no exterior

Do ponto de vista de uma relação comportamental entre os preços da soja cotada no Estado de Maranhão $(P)$, no exterior $\left(P^{*}\right)$, e a taxa de câmbio nominal $(E)$, é notável que há uma correlação positiva (Gráfico 1$)$. A taxa de câmbio nominal $(E)$ exibe um comportamento que se mostra relacionado com as variáveis de preço, entretanto, não acompanha os movimentos de curto prazo. O que se mostra mais evidente é a vantagem dos preços cotados na Bolsa de Chicago em relação aos preços maranhenses, já a partir de 2009. O efeito comportamental dos preços externos sobre o preço doméstico sugere uma possível transmissão assimétrica dos preços com prejuízo aos produtores maranhenses. Os impulsos de crescimento são maiores no preço externo, nos momentos recessivos, o preço doméstico cai mais do que o externo, especialmente no período entre setembro de 2009 e setembro 2014. A ampliação desse gap de preços pode estar relacionada a crise financeira de 2008, e a mudança de destino da maior parte das exportaçóes brasileiras para China. Depois disso a relação se mostra mais estável. Sobre o gap de vantagem dos preços externos, alguns fatores podem explicar, entre eles: a política monetária estadunidense, a especulação financeira, e custos de produção (MEYER; TAUBADEL, 2004). 
Gráfico 1: Preços mensais da soja no mercado internacional e no estado do Maranhão, abril de 2009 a fevereiro de 2016

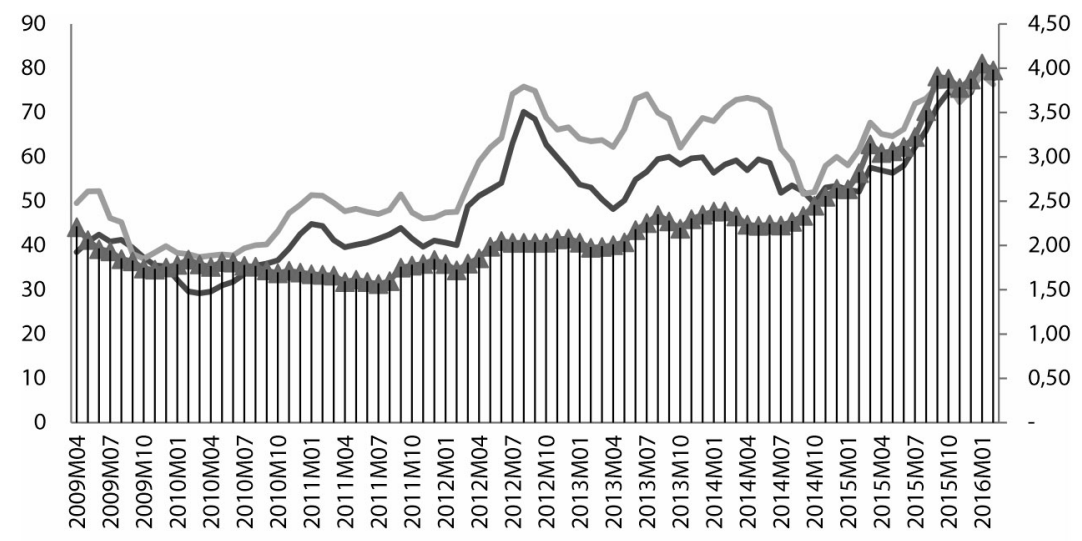

Fonte: Elaboração própria com dados da pesquisa.

Nota: 0 eixo esquerdo reflete os preços da soja no Maranhão $(P)$ e na Bolsa de Chicago $\left(P^{*}\right)$; e 0 eixo direito mede a taxa de câmbio nominal brasileira em Reais (E).

\subsection{Condicionantes do modelo VEC}

O Vetor de Correção de Erros (VEC) pressupõe duas condiçóes estatísticas sobre um as séries temporais, que são: possuir a mesma ordem de integração $(\mathrm{I}(\mathrm{d}))$, e estarem cointegradas ou estáveis no longo prazo. Para verificar esses condicionantes foram realizados os testes de Dickey-Fuller Aumentado (Tabela 1) e de Cointegração de Johansen (Tabela 2). Os referidos testes são usados respectivamente para identificar a ordem de integração e a estabilidade de longo prazo entre as séries da taxa de câmbio nominal brasileira, e dos preços nacional e externo da soja. Por se tratar de um modelo multivariado com três variáveis, a transmissão de preço estimado por um VEC é auxiliado pelo teste de causalidade Granger (Tabela 3), sugerindo a direção da causalidade entre as variáveis de preços do modelo.

Sobre os resultados dos testes de raízes unitárias, claramente se observa que todas as variáveis possuem raiz unitária em nível, mas se tornam estacionárias após o operador de primeira diferença $(\Delta)$. Esses resultados são observados em todas as especificaçóes do teste: passeio aleatório $\left(\tau_{c}\right)$, passeio aleatório com deslocamento $\left(\tau_{c t}\right)$, e passeio aleatório com deslocamento em torno de uma tendência determinística $\left(\tau_{n c}\right)$. Com isso, é possível afirmar que todas as variáveis são integradas de ordem um (I(1)). Portanto, esse resultado sugere que deve haver estabilidade de longo prazo entre essas séries temporais. Isso significa que o uso dessas variáveis em nível não exerce efeito espúrio sobre as estimaçóes, dada a estabilidade de longo prazo (Tabela 1). 
Tabela 1- Teste de raiz unitária Dickey Fuller Aumentado (ADF)

\begin{tabular}{l|c|c|c|c|c|c|l}
\hline Variáveis & $\tau_{c}$ & prob $<\tau_{c}$ & $\tau_{c t}$ & prob $<\tau_{n c}$ & $\tau_{n c}$ & prob $<\tau_{c t}$ & $I(d)$ \\
\hline$P$ & $-0,9179$ & 0,7779 & $-2,9503$ & 0.1528 & 0,7857 & 0,8808 & $\mathrm{I}(1)$ \\
\hline$\Delta P$ & 6,3738 & 0,0000 & -6.3936 & 0,0000 & $-6,3325$ & 0,0000 & $\mathrm{I}(0)$ \\
\hline$P^{*}$ & $-1,2544$ & 0,6471 & $-3,1841$ & 0,0949 & 0,4208 & 0,8020 & $\mathrm{I}(1)$ \\
\hline$\Delta P^{*}$ & $-6,5966$ & 0,0000 & -6.5954 & 0,0000 & $-6,6116$ & 0,0000 & $\mathrm{I}(0)$ \\
\hline$E$ & 1,3556 & 0,9987 & $-1,8822$ & 0,6546 & 2,0994 & 0,9911 & $\mathrm{I}(1)$ \\
\hline$\Delta E$ & $-6,3707$ & 0,0000 & $-6,8582$ & 0,0000 & $-6,1501$ & 0,0000 & $\mathrm{I}(0)$ \\
\hline
\end{tabular}

Fonte: Elaboração própria com dados da pesquisa.

Notas: Valores críticos para $\tau_{c}, \tau_{c t} \tau_{n c}$, obtidos conforme Mackinnon (1996). Os valores críticos adotados nesse trabalho foram em nível de 1\%. A estimativa recursiva One-Step Forecast indica ausência de quebras estruturais no período, portanto, não se fez necessário $0 \mathrm{ADF}$ com quebra estrutural.

Para se certificar dessa ausência de efeitos espúrios o teste de Cointegração de Johansen é realizado usando a especificação recomendada para especificação tendência determinística no intercepto, e no intercepto e tendência permitindo uma especificação de tendência linear determinística (JOHANSEN, 1995). Nesse teste se usa as seguintes hipóteses nulas: não existem vetores de cointegração $(r=0)$, no máximo 1 vetor de cointegração $(r=1)$, e no máximo 2 vetores de cointegração $(r=2)$. De acordo com a probabilidade estatística do teste, apenas a primeira hipótese é rejeitada, de que não existem vetores de cointegração. Nesses termos é possível aceitar a hipótese alternativa de que existe ao menos 1 vetor de cointegração a $5 \%$ de significância. Esse resultado é suficiente para aceitar a hipótese alternativa de que existe ao menos um vetor de cointegração, e que as variáveis do modelo estão em equilíbrio de longo prazo (Tabela 2). A cointegração implica que por mais que o comportamento das series temporais sejam diferentes no curto prazo, no longo prazo, o movimento de uma série em relação a outra é estável. Portanto, dadas as características da relação dinâmica entre os preços, é possível usar o estimador Vetorial de Correção de Erros (ENDERS, 2004; ADAMI; MIRANDA, 2011).

Tabela 2 - Teste de Cointegração de Johansen

\begin{tabular}{l|c|c|c|c}
\hline Rank $(r)$ & $\begin{array}{c}\text { Estatística } \\
\text { Traço }\end{array}$ & Prob. & $\begin{array}{c}\text { Estatística } \\
\text { Máximo autovalor }\end{array}$ & Prob. \\
\hline 0 & 47.53725 & 0.0116 & 30.89714 & 0.0098 \\
\hline 1 & 16.64011 & 0.4423 & 13.22714 & 0.3100 \\
\hline 2 & 3.412964 & 0.8243 & 3.412964 & 0.8243 \\
\hline
\end{tabular}

Fonte: Elaboração própria com dados da pesquisa.

Nota: ** significativo a 5\% com tendência determinística linear. 
Para identificar a fonte de variação entre os preços do modelo de transmissão de preços foi realizado o teste de causalidade Granger. Esse teste sugere uma origem para a fonte de variaçóes nos preços e em que sentido elas são transmitidas. Os resultados indicam que as variações de preços se originam na bolsa de valores de Chicago $\left(P^{*}\right)$ e são transmitidas para os preços doméstica da soja no estado do Maranhão. Esse resultado é identificado quando se rejeita a hipótese $h_{0}$ de que $P_{t-1}^{*}$ não causa $P_{t}$, a $5 \%$ de significância, e não se rejeita a hipótese de que $P_{t-1}$ não causa $P_{t}^{*}$. Esse tipo de causalidade é denominado unidirecional. O fato de $P^{*}$ estar causando o preço doméstico da soja é algo esperado por se tratar do efeito de uma economia grande sobre uma economia pequena, a economia pequena acaba sendo tomadora de preços. Este resultado corrobora outros estudos que avaliam a relação entre preços domésticos versus preços externos (MARGARIDO et al., 2001; MARGARIDO, 2012; LARRE, 2019; PRESOTTO et al., 2018). A causalidade unidirecional do preço externo $\left(P^{*}\right)$ para preço ao produtor no Maranhão $(P)$ significa que variaçóes dos preços da soja na Bolsa de Chicago como proxy do preço internacional precede variaçóes de seus preços no mercado da soja do estado de Maranhão $(P)$.

Tabela 3 - Resultado do teste de causalidade de Granger

\begin{tabular}{l|c|c|l}
\hline Hipótese nula $\left(h_{o}\right)$ & Estatística $\mathrm{F}$ & Probabilidade & \multicolumn{1}{|c}{ Resultado } \\
\hline$P_{t-1}^{*}$ não causa $P_{t}$ & 3.34751 & 0.0236 & Rejeita $h_{o}$ \\
\hline$P_{t-1 \text { não causa } P_{t}^{*}}$ & 1.25719 & 0.2954 & Não rejeita $h_{o}$ \\
\hline$E_{t-1}$ não causa $P_{t}$ & 1.31686 & 0.2755 & Não rejeita $h_{o}$ \\
\hline$P_{t-1 \text { não causa } E_{t}}$ & 1.89077 & 0.1387 & Não rejeita $h_{o}$ \\
\hline$E_{t-1}$ não causa $P_{t}^{*}$ & 1.3291 & 0.2715 & Não rejeita $h_{o}$ \\
\hline$P_{t-1}^{*}$ não causa $E_{t}$ & 0.7007 & 0.5546 & Não rejeita $h_{o}$ \\
\hline
\end{tabular}

Fonte: Elaboração própria com dados da pesquisa.

\section{Estimação do modelo de transmissão de preços}

Dadas as condições de cointegração de series com a mesma ordem de integração apresentadas nas Tabelas (1 e 2), é possível estimar a elasticidade de transmissão dos preços através do estimador VEC sem efeitos espúrios. Entretanto, o VEC é um modelo de defasagens temporais, uma das primeiras etapas da sua estimação é a determinação do número de defasagens ótimas do 
modelo a ser estimado. A determinação dessa defasagem é auxiliada por um conjunto de critérios de ajuste do estimador VEC, que são: Erro de Previsão Final (EPF), Akaike (AIC), Schwarz (SC), e Hannan-Quinn (HQ). Todas essas estatísticas de ajuste apontam que a defasagem ótima para o modelo estimado é de 1 período, a $5 \%$ de significância (Tabela 4).

Tabela 4 - Defasagem ótima do modelo VEC ajustado com base nos critérios de informação FPE, AIC, SC, HQ

\begin{tabular}{l|c|c|c|c}
\hline $\begin{array}{l}\text { Defasagem } \\
\text { ótima }\end{array}$ & EPF & AIC & SC & HQ \\
\hline 1 & $0.073184^{*}$ & $5.898725^{*}$ & $6.174732^{*}$ & $6.009031^{*}$ \\
\hline 2 & 0.078518 & 5.968213 & 6.520228 & 6.188825 \\
\hline 3 & 0.078798 & 5.969409 & 6.797433 & 6.300327 \\
\hline 4 & 0.088715 & 6.083310 & 7.187341 & 6.524534 \\
\hline
\end{tabular}

Fonte: Elaboração própria com dados da pesquisa.

Nota: * Ordem de defasagem selecionada segundo cada critério a 5\% de significância; EPF (Erro de predição final); AIC (Critério de Informação de Akaike); SC (Critério de informação de Schwarz); HQ (Critério de Hannan-Quinn).

A Tabela 5 apresenta as elasticidades de transmissão de preços do mercado internacional de soja para a soja maranhense, usando o estimador do Vetor de Correção de Erros (VEC), sem e com a restrição da Lei do Preço Único. Em ambos os casos, o preço internacional da soja da Bolsa de Valores de Chicago tem efeito positivo sobre o preço da soja maranhense, com significância estatística de 5\%. Esse resultado é coerente com a teoria de transmissão de preços do mercado internacional, e com o teste de causalidade Granger realizado. As demais variáveis do modelo multivariado não mostram significância estatística. Portanto, o câmbio não se mostra relevante na determinação do preço doméstico da soja maranhense, assim como o seu preço não se revela auto-regressivo. Um resultado semelhante pode ser encontrado para o Brasil em Brum et al. (2015). Assim, concentrando-se apenas na transmissão de preços da soja internacional $\left(P^{*}\right)$ para a soja maranhense $(P)$, é possível ver que há uma elasticidade de transmissão no curto prazo $(\alpha)$ de $0.29485 \%$ no modelo irrestrito, e elasticidade de $0.27334 \%$ no modelo restrito a Lei do Preço Único, dado um choque de $1 \%$ no preço externo $\left(P^{*}\right)$. A elasticidade de curto prazo indica a velocidade de ajuste do preço doméstico causado por um choque não anunciado no preço externo da soja. Nesse sentido, o ajuste se mostra ser mais rápido no modelo irrestrito.

Contudo, a velocidade de ajuste pode não ser a melhor referência sobre a transmissão dos preços. Analisando o parâmetro de longo prazo $(\beta)$ do estimador irrestrito, observa-se que a transmissão de preços é de aproxima- 
damente $1.15 \%$ com significância estatística de $1 \%$. Ou seja, é quase possível assumir que há uma transferência integral dos preços internos para o preço doméstico da soja maranhense. No modelo restrito a Lei do Preço Único, que assume por hipótese a transferência integral do preço externo, a ausência de transferência integral dos preços, é rejeitada a $1 \%$ de significância. A estatística $\chi^{2}$ do teste de restrição, indica que a probabilidade de rejeitar a hipótese nula quando ela é falsa é de $0.071 \%$. Na prática, quando condicionada a transferência integral dos preços no longo prazo, o parâmetro $(\alpha)$ de elasticidade passa a expressar uma estimativa mais realista da transmissão internacional do preço da soja. Os parâmetros de longo prazo $(\beta)$ do modelo irrestrito assim como do modelo restrito estão normalizados para o preço doméstico da soja no Maranhão, por isso, são os únicos valores positivos. Como se trata de um modelo multivariado que toma como referência a primeira equação do sistema, o preço doméstico $(P)$ passa a ser considerado endógeno, e as demais variáveis $\left(P^{*}, E\right)$ passam a ser exógenas. Por esse motivo os parâmetros de longo prazo das variáveis exógenas são negativos. Sua análise deve inverter os sinais dos coeficientes, uma vez que estão do mesmo lado da equação de cointegração normalizada.

Os choques não anunciados sobre o preço externo da soja $\left(P^{*}\right)$ têm um efeito positivo no preço da soja doméstica $(P)$ que diferem em intensidade e duração nos modelos irrestrito e restrito. No modelo irrestrito, o qual despreza a hipótese de transmissão integral do preço externo no longo prazo, a intensidade da transmissão aumenta até o terceiro mês, e cai a partir do quarto, do quinto mês em diante se mantem em níveis inferiores ao preço

Tabela 5 - Estimativas de curto e longo prazo do Modelo Vetorial de Correção de Erros sem e com restrição da Lei do Preço Único

\begin{tabular}{|c|c|c|c|c|}
\hline \multirow[b]{2}{*}{ Variáveis } & \multicolumn{2}{|l|}{ Modelo irrestrito } & \multicolumn{2}{|l|}{ Modelo restrito } \\
\hline & $\begin{array}{c}\text { Parâmetros de curto } \\
\text { prazo (a) }\end{array}$ & $\begin{array}{c}\text { Parâmetros de longo } \\
\text { prazo }(\beta)\end{array}$ & $\begin{array}{c}\text { Estimativas a com } \\
\beta \text { restrito }\end{array}$ & $\begin{array}{l}\text { Restrições } \\
\text { sobre } \beta\end{array}$ \\
\hline$P_{t-1}$ & $\begin{array}{r}-0.09197 \\
(0.15275) \\
{[-0.60214]} \\
\end{array}$ & 1.000000 & $\begin{array}{r}-0.07505 \\
(0.149849) \\
{[-0.50088]} \\
\end{array}$ & 4.135121 \\
\hline$P_{t-1}^{*}$ & $\begin{array}{r}0.29485^{* *} \\
(0.11846) \\
{[2.48889]}\end{array}$ & $\begin{array}{r}-1.151538^{* * *} \\
(0.11260) \\
{[-10.2269]}\end{array}$ & $\begin{array}{r}0.27334^{* *} \\
(0.113961) \\
{[2.39859]}\end{array}$ & 4.135121 \\
\hline$E_{t-1}$ & $\begin{array}{r}-0.02782 \\
(0.17440) \\
{[-0.15955]}\end{array}$ & $\begin{array}{r}-0.132878 \\
(0.10312) \\
{[-1.28855]}\end{array}$ & $\begin{array}{c}-0.019807 \\
(0.171764) \\
{[-0.11532]}\end{array}$ & 4.135121 \\
\hline $\begin{array}{l}\text { Teste de } \\
\text { restrição }\end{array}$ & Probability & & $\begin{array}{l}14.48099 \\
0.000717\end{array}$ & \\
\hline
\end{tabular}

Fonte: Elaboração própria com dados da pesquisa.

Nota: ${ }^{* * *},{ }^{* *}$ significância a 1\% e 5\%, com desvio-Padrão entre parênteses e estatística t entre colchetes. 
anterior ao choque, ou seja, não estabiliza após o choque. No modelo restrito, a intensidade da transmissão do choque externo sobre o preço doméstico também cresce até o terceiro mês. Contudo, ele mostra uma ligeira vantagem na intensidade do choque em relação ao modelo irrestrito, e a estabilidade do preço doméstico acontece aproximadamente 15 meses após o choque. Além do ajuste gradual do preço doméstico ao choque no preço externo, o que parece ser mais realista dada a posição de tomador de preço do Maranhão no cenário internacional, o preço estabiliza em torno de zero, após uma longa influência da mudança não anunciada no preço da soja no mercado internacional.

Figura 1: Função impulso resposta de $P$ causado por choques não anunciados em $P^{*}$ nos modelos irrestrito e restrito a Lei do Preço Único
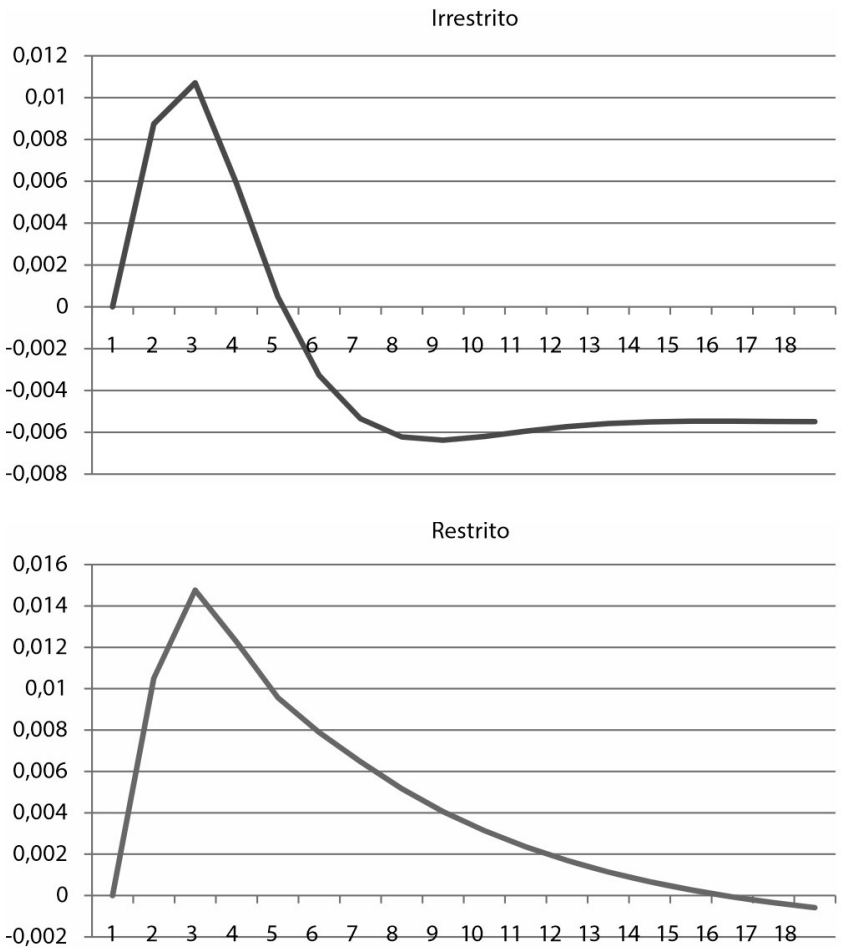

O peso do preço externo da soja $\left(P^{*}\right)$ na decomposição da variância do preço doméstico $(P)$ é maior no modelo restrito do que no modelo irrestrito da estimação. A Tabela 6 mostra que de $100 \%$ da variância do preço doméstico, menos de $3 \%$ se deve ao preço externo do modelo irrestrito, comparati- 
vamente aos mais de $5 \%$ no modelo restrito no terceiro e quarto mês após o choque. No quarto mês, um dos períodos de maior intensidade do choque de transferência de preços nos dois modelos, a variância do preço doméstico devido a um choque no preço externo, no modelo restrito, chega a ser duas vezes a variância devida ao preço externo no modelo irrestrito. Por essas razões, é possível afirmar que existe transferência externa de preço da Bolsa de Chicago para o preço da soja no Maranhão, o mercado de soja maranhense é tomador de preços, há um repasse integral do preço externo para o preço doméstico da soja no longo prazo, e a elasticidade de transferência de preços no curto prazo é de 0.27334 condicionado a Lei do Preço Único.

Tabela 6 - Decomposição da variância do preço da soja no Estado do Maranhão

\begin{tabular}{l|r|r|c}
\hline Período & $P^{*}$ irrestrito & $P^{*}$ restrito & $P^{*}$ restrito $/ P^{*}$ irrestrito \\
\hline 2 & 1.815475 & 2.601618 & 1.433023 \\
\hline 3 & 2.963142 & 5.006427 & 1.689567 \\
\hline 4 & 2.592014 & 5.413183 & 2.088408 \\
\hline & $\ldots$ & $\ldots$ & $\ldots$ \\
\hline 15 & 1.647219 & 2.27879 & 1.383417 \\
\hline
\end{tabular}

Fonte: Elaboração própria com dados da pesquisa. 


\section{CONCLUSÕES}

Essa pesquisa analisa a transmissão de preços no mercado da soja maranhense, sua estabilidade, causalidade direcional, e elasticidade de transmissão. Baseado nesse conjunto de intensões é possível afirmar que há transmissão de preços, essa transmissão é estável no longo prazo, e o preço interno é dependente do preço externo no curto prazo. O preço doméstico da soja maranhense é explicado pela cotação internacional da Soja na Bolsa de Valores de Chicago. Os choques não anunciados no preço externo da soja exercem um efeito positivo sobre o preço da soja do estado de Maranhão. No curto prazo, a intensidade do choque no preço interno cresce até o terceiro mês e estabiliza 15 meses depois. Esse é o período que se mostra necessário para o preço da soja no Maranhão absorver integralmente os choques não anunciados no preço internacional. No longo prazo, a transferência de preço é significativa, e se enquadra na condição de transferência integral dos preços de acordo com a Lei do Preço Único.

O teste de cointegração entre as variáveis da equação empírica rejeita a hipótese de ausência de vetores de cointegração, assim, é possível afirmar que há estabilidade de longo prazo na transferência de preços. $\mathrm{O}$ teste de causalidade rejeita apenas a hipótese de ausência de causalidade do preço externo cotado na Bolsa de Chicago para o preço interno da soja cotada no Maranhão. As demais hipóteses de causalidade do modelo multivariado, não são rejeitadas. Por essa razão, é presumível concluir que é o preço externo da soja que determina o seu preço doméstico no Maranhão. A taxa de cambio não mostra causalidade significativa. As elasticidades de transmissão de preços de curto prazo estimadas através do VEC confirmam que apenas o preço externo da soja se mostra significativo a $5 \%$, para explicar o preço da soja ao produtor, no estado do Maranhão. Entretanto, no longo prazo, não é possível rejeitar a hipótese de que haja transferência integral dos choques exógenos na taxa de câmbio para o preço da soja do maranhão. As elasticidades de transferência de preços estimadas através do VEC restrito, não rejeita a hipótese de transferência integral dos preços externo da soja e cambial. 


\begin{abstract}
Notas
${ }^{1}$ Exemplo mesorregião leste.

${ }^{2}$ Essa variável não foi utilizada neste trabalho, dado que não se pretende tratar de questões relativas à política comercial. Este modelo também não leva em conta diferenças qualitativa entre os produtos, os custos de transporte e armazenagem.

3 Disponível em http://www.agrolink.com.br/cotacoes/historico/ma/soja-em-grao-sc-60kg. Cotações. Acesso em novembro de 2016.

${ }^{4}$ Coletados no site do Fundo Monetário Internacional, International Financial Statistics (FMI/IFS).

${ }^{5}$ As quais são "causadas normalmente por movimentos oscilatórios de mesma periodicidade que ocorrem em período intra-anual, como variações climáticas, férias, feriados, ou outros" (FERREIRA; GONDIN; MATTOS, 2015). Para uma explanação sobre o programa de ajuste sazonal X-12 e X-13 ARIMA, consultar nota técnica da FGV/IBRE, 2015.
\end{abstract}

\title{
Referências
}

Adami, A. C. O. de; Miranda, S. H. G. de. Transmissão de Preços e Cointegração no Mercado Brasileiro de Arroz. RESR. Piracicaba, SP, vol. 49, n. 01, pp. 55-80, jan/ mar 2011.

Brum, Argemiro Luís, Dalfovo, Wylmor Tives, Fernandez, Gilberto Sisto, Zabot, Udilmar Carlos. Análise de co-integração e causalidade entre os mercados regionais de soja no Brasil e os efeitos da taxa de câmbio sobre os preços físicos da commodity soja nas cotações de Sorriso - MT. Organizações Rurais \& Agroindustriais, vol. 17, núm. 3, 2015, pp. 269-284. Universidade Federal de Lavras Minas Gerais, Brasil.

Copetti, Leonardo Sangoi; Coronel, Daniel Arruda. Transmissão da Variação da Taxa de Câmbio para os Preços de Exportação Brasileiros do Grão de Soja. Um Estudo Comparativo do Dólar e do Euro. Desenvolvimento em Questão. Editora Unijuí • ISSN 2237-6453 • Ano 18 • n. 50 • jan./mar. 2020. p. 166-185.

Cunha, Roberto César. Estruturas, estratégias de mercado e comercialização e fluxos de distribuição da soja no sul do Maranhão. XI Encontro Nacional da ANANPEGE. Anais do XI ANANPEGE, 2015. ISSN 2175-8875.

Enders, W. Applied econometric time series. 2.ed. New York: John Wiley \& Sons, 2004.
Ferreira, Costa Pedro; Gondin, Lisboa, José; Mattos, Marcelino Daiane de.

Métodos de ajuste sazonal para séries de Business Tendency: um estudo de caso para a Sondagem da Indústria utilizando o método X13 - ARIMA - SEATA. Norma Técnica FGV IBRE, 2015.

Figueiredo, M. A.; Souza Filho, H. M. de; Paulillo, L. F. O. Análise das Margens e Transmissão de Preços no Sistema Agroindustrial do Suco de Laranja no Brasil. RESR. Piracicaba-SP, vol. 51, n. 2, p. 331-350, abr/jun 2013.

GUANZIROLI, Carlos Enrique.

Metodologia para estudo das relações de mercado em sistemas agroindustriais / Antonio Márcio Buainain; Hildo Meirelles de Sousa Filho - Brasilia: IICA, 2008.

GUJARATI, D. N.; PORTER, D. C. Econometria básica. 5. ed. Editora Porto Alegre, 2011.

IBGE (Instituto Brasileiro de Geografia e Estatística). Produção Agrícola Municipal (PAM). Disponível em www.sidra.ibge.gov. br/tabela/. Capturado em outubro de 2016. . Divisão Territorial Brasileira (2016). [cit. 2016-0115]. https://pt.wikipedia.org/wiki/ Lista_de_mesorregi\%C3\%B5es_e_ microrregi\%C3\%B5es_do_ Maranh\%C3\%A30\#cite_note-IBGE_ DTB_2016-2. 
Instituto Maranhense de Estudos

Socioeconômicos e Cartográficos (IMESC), 2016. Disponível em www.imesc.gov.br. Capturado em 25/10/2016.

JOHANSEN, Soren. Likelihood-based inference in cointegrated vector autoregressive models. New York: Oxford University Press, 1995. 267p. (Advanced Texts in Econometrics).

JOHNSTON, Jack; Dinardo, John. Métodos Econométricos. 4. ed. McGraw-Hill, 2001.

HARRIS, Richard I.D. Cointegration analysis in econometric modelling. London: Prentice Hall, 1995. 176p.

KRUGMAN, Paul. Economia Internacional: teoria e política. São Paulo: Pearson Prentice Hall, 2010.

Larre, Guillermo Andrés. Market Integration in the International Market of Soybeans: Are GM Soy and NonGM Soy Markets Integrated? Journal of Agricultural Science; Vol. 11, No. 15; 2019. ISSN 1916-9752 E-ISSN 1916-976o. Published by Canadian Center of Science and Education.

MAYORGA, O. R; Khan, Ahmad. Saeed; Mayorga, Ruben. Dario; Lima, Patrícia V. P. S; Margarido, M. A. Análise de transmissão de preços do mercado atacadista de melão do Brasil. RESR. Rio de Janeiro, vol. 45, $\mathrm{n}^{\circ}$ 03، p. 675-704, jul/set 2007 - Impressa em agosto 2007.
MARGARIDO, M. A; TUROLLA, F. A.; FERNANDES, J. M. Análise da elasticidade de transmissão de preços no mercado internacional de soja. Pesquisa e Debate. V. 12, n. 2, p. 5-40, 2001.

MARGARIDO, M. A. Análise da transmissão espacial de preços no mercado internacional da soja. Revista de Economia e Administração. V. 11, n. 3, 281-303 pg, jul/ set. 2012.

MEYER, Jochen.; Von. TAUBADEL, C. S. Asymmetric price transmission: a survey. Journal of Agricultural Economics. V. 55, n. 3, p. 581-611, 2004. MUNDLAK, Y; LARSON, D. F. On the transmission of world agricultural prices. The World Bank Economic Review. V.6, n.3, p. 399-422, 1992.

Presotto, E; Freitas, C. A. de; Lazaretti, L. R; Batistella, P; Feistel, P. R. Análise da transmissão do preço pago ao produtor de soja brasileiro (1997 a 2016). Perspectiva Econômica, vol. 14, n. 2, p. 108-122, julhodezembro 2018.

RAVALLION, M. Testing market integration. American Journal of Agricultural Economics, v. 68, n.1, p. 102109, Feb. 1986.

Silva, J. S; Freitas, C. O; Lima, J. E. Integração de preços no mercado da soja nos estados do Mato Grosso, Paraná e Rio Grande do Sul. Economia-Ensaios, Uberlândia, 32 (1): 65-84, Jul./Dez. 2017.

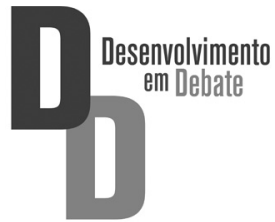

